\title{
Flow cytometric analysis of normal and reactive spleen
}

\author{
Adriana I Colovai ${ }^{1}$, Christina Giatzikis ${ }^{1}$, Eric K Ho ${ }^{1}$, Mushahid Farooqi ${ }^{1}$, \\ Nicole Suciu-Foca ${ }^{1}$, Giorgio Cattoretti ${ }^{1}$ and Attilio Orazi ${ }^{2}$ \\ ${ }^{1}$ Department of Pathology, Columbia University, New York, NY, USA and ${ }^{2}$ Department of Pathology and \\ Laboratory Medicine, School of Medicine, Indiana University, Indianapolis, IN, USA
}

\begin{abstract}
Spleen is surgically removed for both non-neoplastic and neoplastic pathologies. A significant proportion of splenectomy specimens require distinguishing between reactive and neoplastic conditions (eg lymphoma). To establish a 'normal' reference range for the spleen lymphocyte subsets, fresh samples of benign, reactive spleens obtained from adult patients $(N=12)$ and samples of normal spleen obtained from cadaveric transplant donors $(N=14)$ were analyzed using three- and four-color flow cytometry. Study of pan-B, -T, and -NK marker expression revealed that the frequency of $T$ cells is higher and that of $B$ cells is lower in reactive (nonneoplastic) compared to normal (cadaveric) spleen. Furthermore, our study established a frame of reference for cell markers commonly used for immunophenotyping of lymphoma, and identified discrete lymphocyte subsets, such as early plasma cells and T cells carrying the phenotype of the NK/T subset. These results will facilitate an accurate interpretation of the flow cytometric analysis of human spleen lymphocytes.
\end{abstract}

Modern Pathology (2004) 17, 918-927, advance online publication, 23 April 2004; doi:10.1038/modpathol.3800141

Keywords: spleen; flow cytometry; reference range

Flow cytometry is a sensitive technique currently employed by pathologists for quantitative and qualitative evaluation of hematopoietic cells. ${ }^{1-5}$ In a clinical setting, the main purpose of flow cytometric analysis is to identify abnormal cells by defining their immunophenotypic characteristics. To accomplish this task, multiparameter analysis represents a reliable approach that integrates the information provided by forward scatter (FSC) and side scatter (SSC) with multiple fluorescence parameters. Thus, the cell size and internal complexity as well as the simultaneous expression of 3-4 different cell antigens are evaluated and used to outline distinct cell populations. Importantly, the multiparameter analysis permits the detection of abnormal cells that occur at very low frequency in the context of normal populations, such as in the case of minimal residual disease. ${ }^{6,7}$

Three-color flow cytometry is currently used by many laboratories for the analysis of leukemia and lymphoma, although technology is moving rapidly towards more complex types of analysis, employing

Correspondence: Dr AI Colovai, PhD, College of Physicians and Surgeons of Columbia University, 630 W168 Street, P\&S 14-402, New York, NY 10032, USA.

E-mail: aic4@columbia.edu

Received 22 October 2003; revised 24 February 2004; accepted 25 February 2004; published online 23 April 2004 six or more fluorescence parameters. ${ }^{8}$ In three-color analysis, a widely used strategy for the gating of lymphocytes is based on CD45 expression and SSC characteristics. $^{9,10}$ This approach permits the analysis of aged samples with a better lymphocyte recovery compared to FCS vs SSC gating. Therefore, gating by CD45/SSC represents the strategy of choice for immunophenotyping blood lymphocyte subsets, for example, monitoring CD4 counts in AIDS patients. ${ }^{9}$ This strategy is also successfully applied for the analysis of many lymphoid malignancies detected in blood, bone marrow, and lymph nodes. ${ }^{1,9}$

Specific immunophenotypic profiles have been established for several lymphoid neoplasms. ${ }^{1-4}$ The abnormal profiles are compared to the pattern of cell marker expression normally seen in the organ/tissue being examined, and a diagnostic interpretation is made. While the normal reference ranges for differentiation antigens expressed by lymphoid cells from peripheral blood, bone marrow, and lymph nodes are well established, ${ }^{11-15}$ there is little information concerning the immunophenotypic profile of lymphocytes found in normal or reactive spleen. The aim of our study was to provide a detailed immunophenotypic characterization of human spleen lymphocytes using three- and four-color flow cytometry. Our analysis established reference values for commonly used lymphoid markers and 
defined discrete lymphocyte subsets in normal (cadaveric) and reactive (non-neoplastic) spleen.

\section{Material and methods}

Human spleen specimens were obtained from 11 males and 15 females, with an age range of 17-79 years (mean age 42). The specimens included 14 normal spleens obtained from cadaveric organ donors and 12 spleens obtained from patients with non-neoplastic conditions, such as idiopathic thrombocytopenic purpura (ITP) (six cases), splenic infarct (three cases), benign vascular malformations (two cases), and pancreatic fistula (one case). The diagnosis of reactive spleen was established by two experienced pathologists (AO and GC) based on morphology findings. All spleen samples were processed for flow cytometric analysis within $8 \mathrm{~h}$ from harvesting, using a simple and rapid procedure. The tissue was first teased with forceps into RPMI 1640 cell culture medium (MediaTech, Herndon, VA, USA), allowed to settle, and large debris was removed. The cell suspension was then washed and resuspended in phosphate-buffered saline (PBS) supplemented with $2 \%$ fetal calf serum (FCS) and $0.1 \%$ sodium azide. Cell viability was assessed using Trypan Blue. All specimens included in the study showed lymphocyte viability higher than $80 \%$.
To compare the frequency of B-cell subsets identified in the spleen and that found in peripheral blood or lymph nodes, 11 samples of blood and 8 lymph nodes obtained from cadaveric organ donors were included in this study. Lymph nodes were first chopped into small pieces and the resulting cell suspension was washed and resuspended in PBS supplemented with $2 \%$ FCS and $0.1 \%$ sodium azide. Lymph nodes with viability higher than $80 \%$ were included in the study.

\section{Flow Cytometry}

For three-color flow cytometric analysis, aliquots of $5 \times 10^{5}$ cells/tube were stained with saturating amounts of fluorescein isoththiocyanate (FITC)-, phycoerythrin (PE)-, and peridinin chlorophyll protein (PerCP)-conjugated antibodies, according to the manufacturer's instructions. As shown in Table 1, the antibody panel included monoclonal and polyclonal antibodies. CD45 antibody labeled with the PerCP fluorochrome was added to each tube for lymphocyte gating. To minimize the level of nonspecific binding of antibodies used for the detection of immunoglobulin (Ig) light and heavy chains, cells were washed three times in PBS supplemented with $10 \%$ FCS and $0.1 \%$ sodium azide prior to staining. ${ }^{16}$ For intracellular staining, cells were fixed and permeabilized using Fix and

Table 1 Antibody panel

\begin{tabular}{|c|c|c|}
\hline CD marker (FITC/PE/PerCP) & Clone (FITC/PE/PerCP) & Source (FITC/PE/PerCP) \\
\hline CD2/CD19/CD45 & 39C1.5/J4119/2D1 & Beckman Coulter ${ }^{\mathrm{a}} /$ Beckman Coulter/BD \\
\hline CD3/CD19/CD45 & SK7/4G7/2D1 & $\mathrm{BD} / \mathrm{BD} / \mathrm{BD}$ \\
\hline CD4/CD8/CD45 & SK3/SK1/2D1 & BD/BD/BD \\
\hline CD20/CD5/CD45 & L27/L17F12/2D1 & $\mathrm{BD} / \mathrm{BD} / \mathrm{BD}$ \\
\hline CD7/CD13/CD45 & 4H9/L138/2D1 & $\mathrm{BD} / \mathrm{BD} / \mathrm{BD}$ \\
\hline CD10/CD20/CD45 & W8E7/L27/2D1 & $\mathrm{BD} / \mathrm{BD} / \mathrm{BD}$ \\
\hline CD3/CD16+56/CD45 & SK7/B73.1/2D1 & BD/BD/BD \\
\hline Kappa/Lambda/CD45 & TB28-2/1-155-2/2D1 & $\mathrm{BD} / \mathrm{BD} / \mathrm{BD}$ \\
\hline cy Kappa/cy Lambda/CD45 & TB28-2/1-155-2/2D1 & $\mathrm{BD} / \mathrm{BD} / \mathrm{BD}$ \\
\hline TCR alpha/beta/TCR gamma/delta/CD45 & $\mathrm{WT} 31 / 11 \mathrm{~F} 2 / 2 \mathrm{D} 1$ & $\mathrm{BD} / \mathrm{BD} / \mathrm{BD}$ \\
\hline $\mathrm{IgM} /-/ \mathrm{CD} 45$ & Polyclonal/-/2D1 & BioSource International ${ }^{\mathrm{c}} /-/ \mathrm{BD}$ \\
\hline IgG/-/CD45 & Polyclonal/-/2D1 & BioSource International/-/BD \\
\hline IgA/-/CD45 & Polyclonal/-/2D1 & BioSource International/-/BD \\
\hline IgD/-/CD45 & Polyclonal/-/2D1 & BioSource International/-/BD \\
\hline CD103/-/CD45 & $2 \mathrm{G} 5 /-/ 2 \mathrm{D} 1$ & Beckman Coulter/-/BD \\
\hline FMC7/-/CD45 & FMC7/-/2D1 & Beckman Coulter/-/BD \\
\hline cy TdT/-/CD45 & Polyclonal/-/2D1 & Beckman Coulter/-/BD \\
\hline$-/ \mathrm{CD} 11 \mathrm{c} / \mathrm{CD} 45$ & -/S-HCL-3/D1 & $-/ \mathrm{BD} / \mathrm{BD}$ \\
\hline -/CD23/CD45 & -/EBVSC-5/2D1 & $-/ \mathrm{BD} / \mathrm{BD}$ \\
\hline$-/ C D 30 / C D 45$ & -/Ber-H83/2D1 & $-/ \mathrm{BD} / \mathrm{BD}$ \\
\hline -/CD34/CD45 & $-/ 8 \mathrm{G} 12 / 2 \mathrm{D} 1$ & $-/ \mathrm{BD} / \mathrm{BD}$ \\
\hline -/CD38/CD45 & -/HB7/2D1 & $-/ \mathrm{BD} / \mathrm{BD}$ \\
\hline -/HLA-DR/CD45 & -/L243/2D1 & $-/ \mathrm{BD} / \mathrm{BD}$ \\
\hline -/cy CD79a/CD45 & $-/ \mathrm{HM} 47 / 2 \mathrm{D} 1$ & $-/ \mathrm{BD} / \mathrm{BD}$ \\
\hline
\end{tabular}

${ }^{\mathrm{a} B e c k m a n}$ Coulter, Miami, FL, USA.

${ }^{\mathrm{b}} \mathrm{BD}$ BioSciences, San Jose, CA, USA.

${ }^{\mathrm{C}}$ BioSource International, Inc., Camarillo, CA, USA.

cy, cytoplasmic 
Perm reagents (Caltag Laboratories, Burlingame, CA, USA), and then incubated with specific antibodies, as indicated in Table 1. Following staining, all aliquots were subjected to red cell lysis using FACSLyse solution (BD Biosciences, San Jose, CA, USA). The presence of at least 90\% CD45-positive cells within all acquired events was considered indicative of efficient red blood cell lysis.

To study the immunoprofile of splenic B cells, four-color flow cytometry was performed. Splenocytes were stained with the following antibody combinations: CD20 FITC/Kappa PE/CD45 PerCP/ CD19 APC (APC: allophycocyanine), CD20 FITC/ Lambda PE/CD45 PerCP/CD19 or isotype matched control antibodies (BD Biosciences). To test the expression of CD38 and CD138 markers, typically expressed by plasma cells, splenocytes were stained with CD20 FITC/CD38 PE/CD45 PerCP/CD19 APC (BD Biosciences) and CD20 FITC (BD Biosciences)/ CD138 PE (Serotec Inc., Raleigh, NC, USA)/CD45 PerCP/CD19 APC (BD Biosciences).

To compare B-cell populations found in spleen, whole blood and lymph nodes, splenocytes, whole blood and lymphocytes isolated from lymph nodes were stained with CD20 FITC/CD45 PerCP/CD19 APC or isotype matched control antibodies (BD Biosciences). For this, $50 \mu \mathrm{l}$ of whole blood or $5 \times 10^{5}$ lymphocytes isolated from lymph nodes were incubated with each of the indicated antibody mix, then lysed and washed.

Samples were run and analyzed on a FACSCalibur instrument (BD Biosciences). The sensitivity of the fluorescence detectors was set daily using Calibrite beads (BD Biosciences). FSC and SSC measurements were made using linear amplifiers, whereas fluores- cence measurements were made with logarithmic amplifiers. Flow cytometric two-parameter dot plots and quadrant statistics were generated using Cellquest software (BD Biosciences).

\section{Statistical Analysis}

The mean and standard deviation (s.d.) values for percent positive cells were calculated as described. ${ }^{11}$ Student's $t$-test was used to compare the frequency of lymphocyte subpopulations in normal (cadaveric) vs reactive (non-neoplastic) spleens. Matched $t$-test was used to assess the differences between paired results obtained for B-cell subsets. The correlation between age progression and the frequency of lymphocyte subpopulations was determined by simple linear equation using BMPD statistical software. ${ }^{17}$

\section{Results}

The immunophenotypic profile of human spleen lymphocytes was studied using three- and fourcolor flow cytometry. The spleen specimens were obtained from organ cadaveric donors (normal group) and from patients with nonmalignant conditions that required splenectomy and showed minimal involvement of the spleen (reactive group). The antibodies used in this study recognize pan-T, -B, -natural killer (NK), and myeloid/monocytic antigens, covering markers that are essential for evaluation of lymphoma (Table 1). ${ }^{18}$ In addition, the antibody panel included markers that allow a
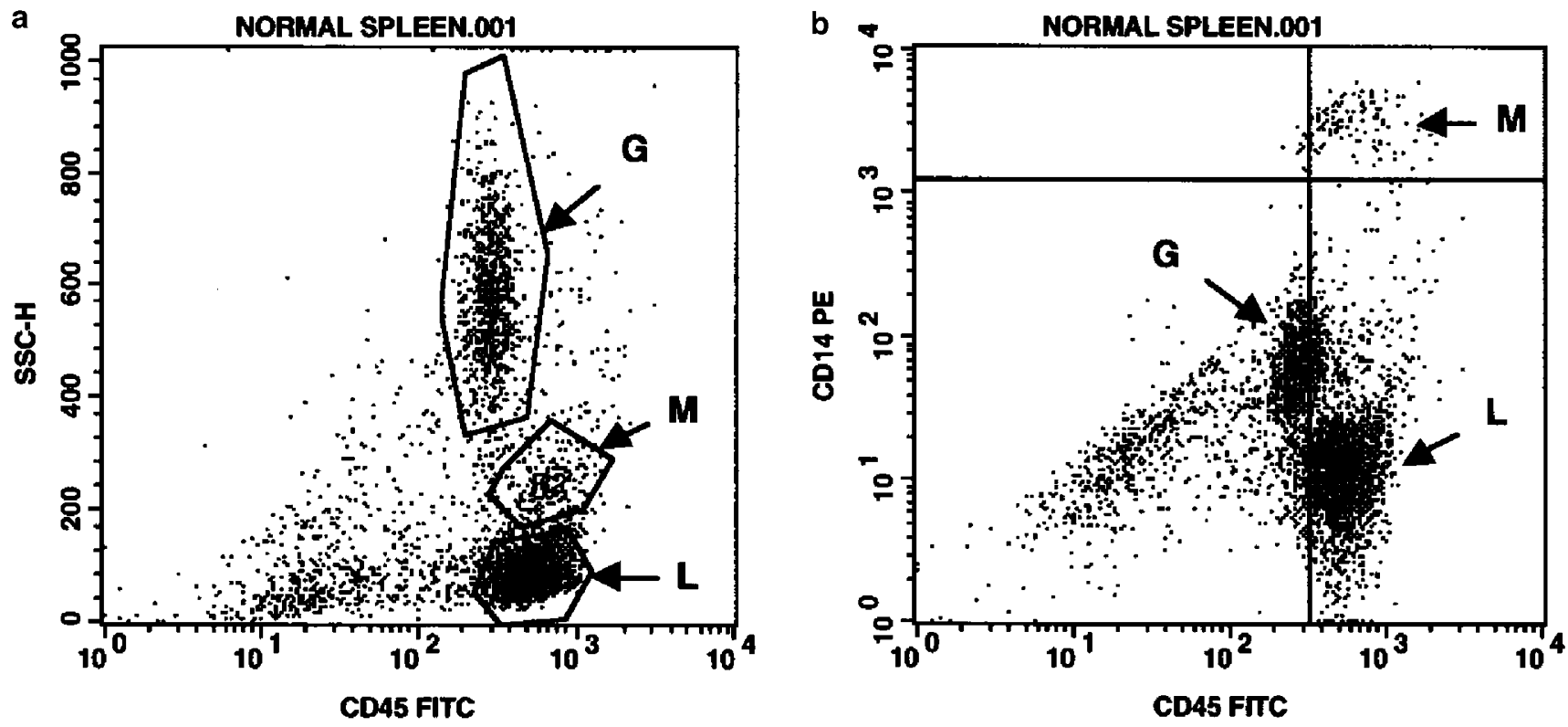

Figure 1 Gating of human spleen lymphocytes. (a) Lymphocytes (L), monocytes (M), and granulocytes (G) from normal spleen were gated using the display of CD45 ( $x$-axis) vs SSC, (y-axis). (b) Spleen monocytes were identified based on the bright expression of CD45 and CD14 markers. 
Table 2 Expression of surface and intracellular markers by human spleen lymphocytes

\begin{tabular}{|c|c|c|c|c|c|}
\hline \multirow[t]{2}{*}{ Cell marker } & \multicolumn{2}{|c|}{$\begin{array}{c}\text { Normal } \\
\text { (cadaveric) } \\
\text { spleen }\end{array}$} & \multicolumn{2}{|c|}{$\begin{array}{c}\text { Reactive } \\
\text { (non-malignant) } \\
\text { spleen }\end{array}$} & \multirow[t]{2}{*}{ P-value } \\
\hline & $N$ & $\underset{(\%)}{\operatorname{Mean} \pm s . d .}$ & $N$ & $\underset{(\%)}{\operatorname{Mean} \pm s . d .}$ & \\
\hline CD2 & 14 & $38 \pm 10$ & 12 & $50 \pm 14$ & 0.015 \\
\hline CD3 & & $31 \pm 9$ & & $43 \pm 14$ & 0.009 \\
\hline CD4 & & $17 \pm 8$ & & $23 \pm 7$ & NS \\
\hline CD5 & & $32 \pm 8$ & & $42 \pm 13$ & 0.028 \\
\hline CD7 & & $37 \pm 11$ & & $44 \pm 12$ & NS \\
\hline CD8 & & $14 \pm 5$ & & $19 \pm 7$ & 0.028 \\
\hline CD4/CD8 ratio & & $1.2 \pm 3$ & & $1.2 \pm 0.2$ & NS \\
\hline CD10 & & $1 \pm 1$ & & $1 \pm 1$ & NS \\
\hline CD11c & & $28 \pm 10$ & & $25 \pm 10$ & NS \\
\hline CD13 & & $1 \pm 1$ & & $1 \pm 1$ & NS \\
\hline CD16/56+CD3- & & $15 \pm 7$ & & $12 \pm 5$ & NS \\
\hline CD16/56+CD3+ & & $5 \pm 2$ & & $5 \pm 4$ & NS \\
\hline CD19 & & $55 \pm 11$ & & $45 \pm 14$ & 0.04 \\
\hline CD20 & & $49 \pm 9$ & & $42 \pm 12$ & NS \\
\hline CD20+CD5+ & & $8 \pm 6$ & & $11 \pm 7$ & NS \\
\hline CD23 & & $34 \pm 13$ & & $35 \pm 12$ & NS \\
\hline CD30 & & $1 \pm 1$ & & $1 \pm 1$ & NS \\
\hline CD34 & & $2 \pm 2$ & & $2 \pm 1$ & NS \\
\hline CD38 & & $79 \pm 13$ & & $83 \pm 12$ & NS \\
\hline CD103 & & $2 \pm 1$ & & $2 \pm 1$ & NS \\
\hline су CD79a & & $52 \pm 12$ & & $44 \pm 10$ & NS \\
\hline HLA-DR & & $71 \pm 11$ & & $71 \pm 12$ & NS \\
\hline $\operatorname{IgM}$ & & $42 \pm 14$ & & $40 \pm 17$ & NS \\
\hline $\mathrm{IgG}$ & & $5 \pm 5$ & & $3 \pm 2$ & NS \\
\hline IgA & & $9 \pm 7$ & & $6 \pm 2$ & NS \\
\hline IgD & & $36+12$ & & $35+12$ & NS \\
\hline FMC7 & & $38 \pm 13$ & & $30 \pm 10$ & NS \\
\hline Карра & & $28 \pm 5$ & & $25 \pm 8$ & NS \\
\hline Lambda & & $21 \pm 5$ & & $17 \pm 6$ & NS \\
\hline Kappa/Lambda & & $1.3 \pm 0.2$ & & $1.4 \pm 0.2$ & NS \\
\hline cy Kappa & & $31+6$ & & $29+8$ & NS \\
\hline cy Lambda & & $22 \pm 5$ & & $16 \pm 6$ & NS \\
\hline cy TdT & & $<1$ & & $<1$ & NS \\
\hline TCR alpha/beta & & $29 \pm 9$ & & $37 \pm 12$ & NS \\
\hline TCR gamma/delta & & $2 \pm 2$ & & $5 \pm 1$ & NS \\
\hline
\end{tabular}

${ }^{\mathrm{a}} \mathrm{NS}$, not significant $(P>0.05)$.

detailed analysis of abnormal cells, beyond the initial evaluation of the case.

As indicated in Figure 1, spleen lymphocytes were gated based on bright expression of the CD45 marker and low SSC. Among the nucleated cells, lymphocytes accounted in average for $82 \%$ of the cells. Monocytes were identified in each sample using the CD14 antibody, and were found to represent about $2 \%$ of all nucleated cells. The mean percentage and s.d. values for each marker or combination of markers analyzed within the lymphocyte gate are indicated in Table 2 .

The comparison between normal and reactive spleen using the Student's $t$-test of significance indicated that, for several T- and B-cell markers, the differences between cell frequencies found in the two groups of specimens were statistically significant. Thus, the frequency of lymphocytes expressing CD2, CD3, CD5, or CD8 was significantly higher in reactive compared to normal spleen, while the frequency of CD19-positive cells was lower in reactive vs normal spleen (Table 2). The mean percentages of cells expressing other T-cell markers, such as CD4 or T-cell receptor (TCR), were also higher in reactive vs reactive spleen, although the differences did not reach statistical significance. Likewise, the mean percentages of lymphocytes expressing pan-B-cell markers, such as CD20, FMC7, CD79a, heavy and light Ig chains, were lower in reactive compared to normal spleen. The frequency of NK cells appeared only slightly increased in normal vs reactive spleen.

\section{B-cell Profile}

A typical immunoprofile of spleen B lymphocytes obtained by three-color analysis is indicated in Figure 2. The lymphocytes were gated using the CD45/SSC display (Figure 1) and analyzed for the expression of the following markers: CD19, CD20, FMC7, cytoplasmic CD79a, surface and cytoplasmic Kappa and Lambda Ig chains, surface IgA, IgD, IgG and IgM chains, HLA-DR, CD10, and CD23. As shown in Table 2, the frequency of CD19 and cytoplasmic CD79a-positive cells had mean values of 55 and $52 \%$, respectively, in normal spleens, and 45 and $44 \%$, respectively, in reactive spleen. Student's $t$-test of significance indicated that there were no statistical differences between the frequency of CD19 and cytoplasmic CD79a-positive cells within each group $(P>0.05)$. However, a statistically significant difference was found when the frequency of cells expressing CD19 was compared with the frequency of CD20 or FMC7 expressing cells in paired samples of normal (cadaveric) spleen. Thus, CD20 was found on $49 \%(P=0.01)$, while FMC7 was present on $38 \%(P<0.001)$ of the lymphocytes in normal spleen. In reactive spleen, the difference between the frequencies of cells expressing CD19 and CD20 did not reach statistical significance, yet the difference between CD19 and FMC7 expressing cells was significant $(P<0.001)$.

The percentage of the lymphocytes expressing Kappa and Lambda Ig light chains was $49 \%$ in normal spleen, being significantly lower than the percentage of CD19 positive cells $(P<0.01)$ and matching the frequency of CD20-positive cells $(P>0.05)$. In reactive spleen, the frequency of the lymphocytes expressing Ig light chains was also lower than that of the cells expressing the CD19 marker, although the difference was not statistically significant. Notably, the frequency of lymphocytes displaying both surface and cytoplasmic expression of Kappa and Lambda chains matched closely the percentage of CD19-positive cells in normal (53\%) and reactive $(45 \%)$ spleen, respectively $(P>0.05)$.

To further define the phenotypic profile of splenic B cells, four-color flow cytometric analysis was performed. As shown in Figure 3, nearly all CD19 + CD20 + cells were positive for either surface 

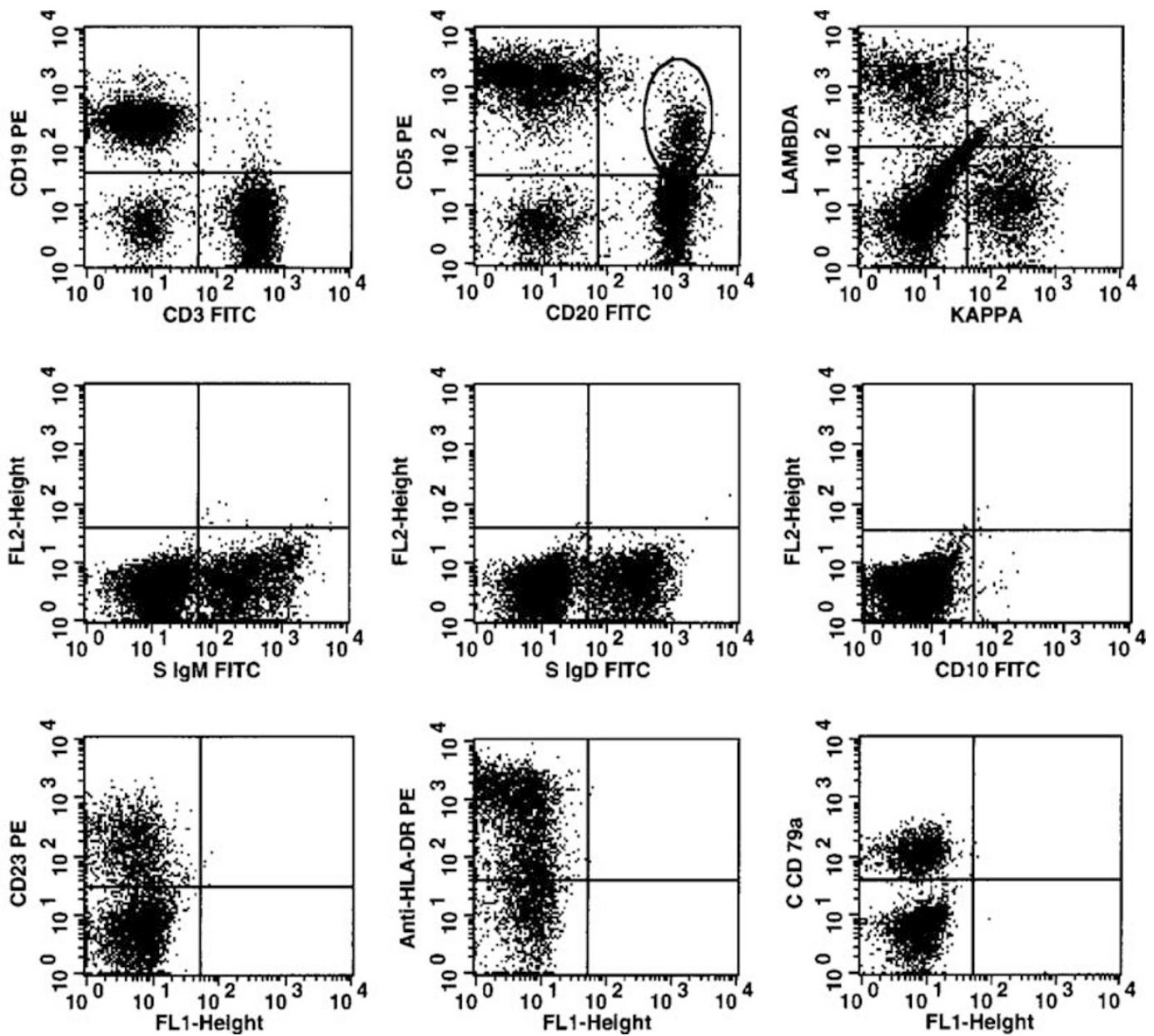

Figure 2 Immunophenotypic profile of splenic B cells. Human spleen lymphocytes were gated as indicated in Figure 1 and studied for expression of pan-B-cell markers by three-color flow cytometry. The oval highlights the population of CD20-positive B cells coexpressing the CD5 marker.

Kappa or Lambda light chain. However, a distinct population carrying the CD19+CD20- phenotype and accounting for 6 and $3 \%$ of all CD19-positive cells in normal and reactive spleen, respectively, showed very dim or no expression of Kappa and Lambda Ig light chains. To determine whether these B cells represent plasma cells, expression of CD38 and CD138 was analyzed by four-color flow cytometry. As illustrated in Figure 4a, CD19 + CD20- B cells showed expression of high levels of CD38 yet were negative for CD138. These results are consistent with the phenotype of early plasma cells. ${ }^{19-21}$

To compare the frequency of CD19+CD20- B cells from human spleen and that from normal peripheral blood or lymph nodes, 11 samples of blood and 8 lymph nodes were analyzed by threecolor flow cytometry for expression of CD45, CD20, and CD19. The frequency of CD19+CD20- B cells was lower than $1 \%$ in peripheral blood and reached a mean of $1 \%$ in lymph nodes (Figure $4 \mathrm{~b}$ ). Thus, CD19+CD20- B cells appear to be more abundant in spleen as compared to peripheral blood or lymph nodes.
A significant subset of splenic B cells, representing 8 and 11\% of the lymphocytes (16 and $26 \%$ of CD20-positive cells) in normal and reactive spleen, respectively, was found to coexpress the CD20 and CD5 markers (Figure 2, Table 2). As illustrated in Figure 2, the level of CD5 expression on B cells was typically one log less compared to the level seen on T cells (CD5 + CD20-) in any of the spleen samples. CD5 + B cells have been previously identified as the B-1a subset and shown to represent a major subset during fetal life and childhood. ${ }^{22}$ To determine whether age-related changes in the frequency of CD5 + CD20 + cells occur in the spleen, the distribution of cell frequency values vs donor's age was studied using simple linear equation. As shown in Figure 5, there was a statistically significant decrease in the percent of CD5 + CD20 + B cells with age $(P<0.02)$. The relationship between the frequency of other lymphocyte subsets, as defined by the expression of CD3, CD19, CD20, CD16/56, or CD23 marker, and donor's age was also studied. No significant correlation was found for any of these cell subsets (data not shown). 


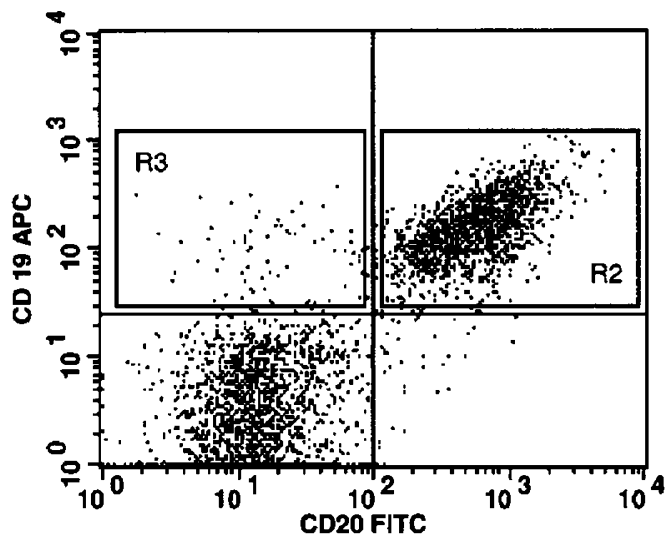

\begin{tabular}{crrr} 
Gate: G1 & \multicolumn{3}{c}{ Gated Events: 3652} \\
Quad \% & Gated & X Mean & Y Mean \\
\hline UL & 2.57 & 33.47 & 97.10 \\
UR & 40.47 & 707.14 & 189.04 \\
LL & 55.81 & 17.62 & 4.08 \\
LR & 1.15 & 253.86 & 12.46
\end{tabular}
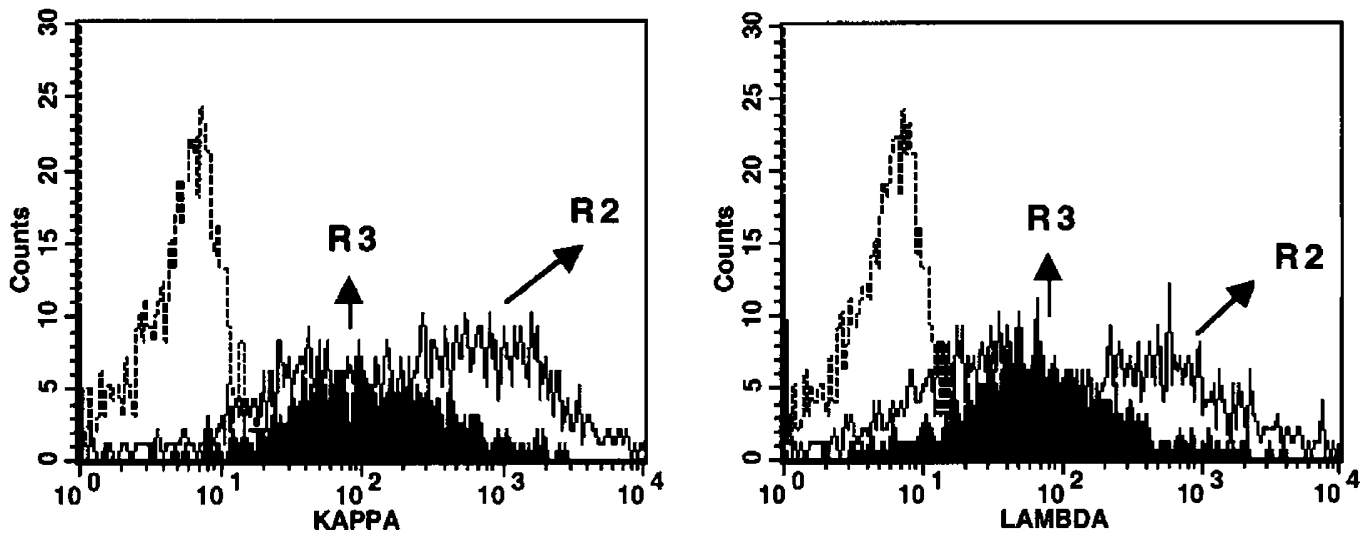

Figure 3 Expression of Kappa and Lambda Ig light chains by splenic B-cell subsets. Spleen lymphocytes were gated as indicated in Figure 1 and analyzed by four-color flow cytometry using CD20/Kappa/CD45/CD19 and CD20/Lambda/CD45/CD19 antibody cocktails. CD19 + CD20 + (gate R2) and CD19 + CD20 - (gate R3) B-cell subsets were studied for expression of Kappa and Lambda Ig light chains, as illustrated by the histograms. Dotted lines indicate staining with isotype matched antibody used as negative control.

CD10 (CALLA), a characteristic marker of germinal center B cells and a hallmark of follicular lymphoma, ${ }^{5,23}$ was expressed on a small number of cells from normal (mean 1\%) and reactive (mean $2 \%$ ) spleens. CD103, a useful marker for the diagnosis of hairy cell leukemia, ${ }^{7}$ was expressed at similarly low frequency. Markers that are frequently expressed by immature cells, such as CD34 or TdT, were found on $2 \%$ or less of the spleen lymphocytes (Table 2).

\section{T and NK Cell Profile}

A typical T-cell profile of spleen lymphocytes is depicted in Figure 6. Pan-T cell markers CD2 and CD3 were expressed on 38 and $31 \%$, respectively, of normal spleen lymphocytes, and on 47 and $40 \%$, respectively, of reactive spleen cells. Staining with antibodies specific for TCR $\alpha / \beta$ and $\gamma / \delta$ chains indicated that the $\alpha / \beta$ heterodimer was expressed on $29 \%$ of lymphocytes from normal spleen and $34 \%$ of lymphocytes isolated from reactive spleen, while the $\gamma / \delta$ chains were present on only $2 \%$ of lymphocytes from normal spleen and on $4 \%$ of lymphocytes from reactive spleen. The NK-specific markers CD16/CD56 were expressed in the absence of CD3 on 15 and $12 \%$ of the lymphocytes from normal and reactive spleen, respectively, and coexpressed with CD3 on 5\% of lymphocytes in both groups (Figure 6, Table 2). CD30, a useful marker for the diagnosis of anaplastic large cell lymphoma ${ }^{24}$ was virtually absent on normal splenic lymphocytes.

\section{Discussion}

The spleen is the largest lymphoid tissue of the human body, accounting for approximately $25 \%$ of the total number of lymphocytes. To establish the immunophenotypic profile of lymphocyte subsets found in human spleen, we performed three- and four-color flow cytometric analysis of the cells isolated from fresh spleen tissue obtained from cadaveric donors and patients with nonmalignant conditions. The extensive antibody panel used in this study allowed a detailed characterization of the lymphocyte subsets found in normal and reactive spleen. The two groups of specimens showed significantly different frequencies of CD2, CD3, CD5, CD8, and CD19 expressing cells. These results 


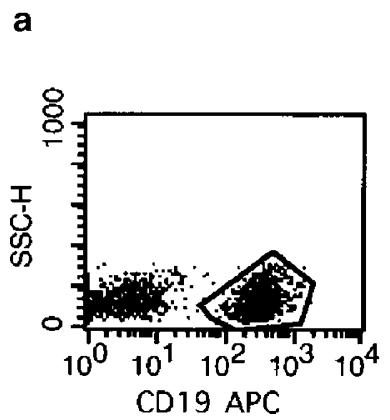

b
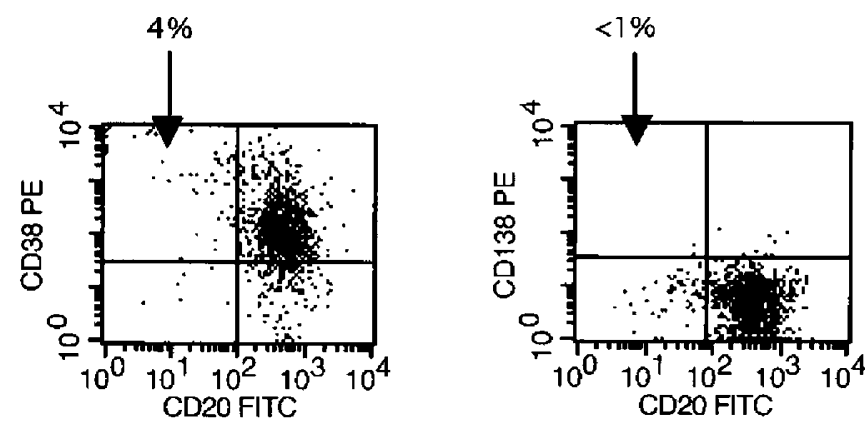

PERIPHERAL BLOOD
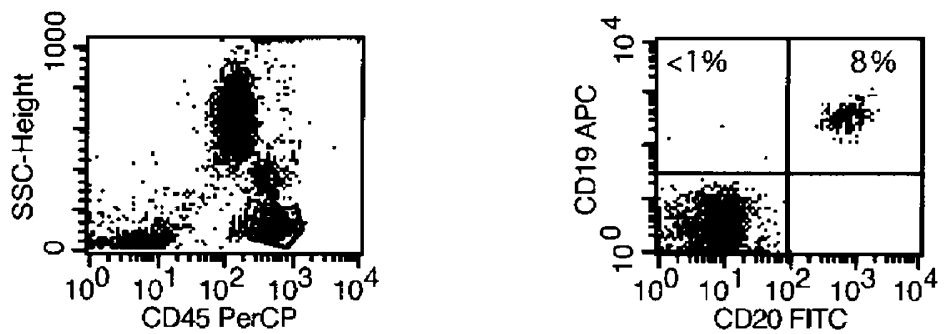

\section{LYMPH NODE}
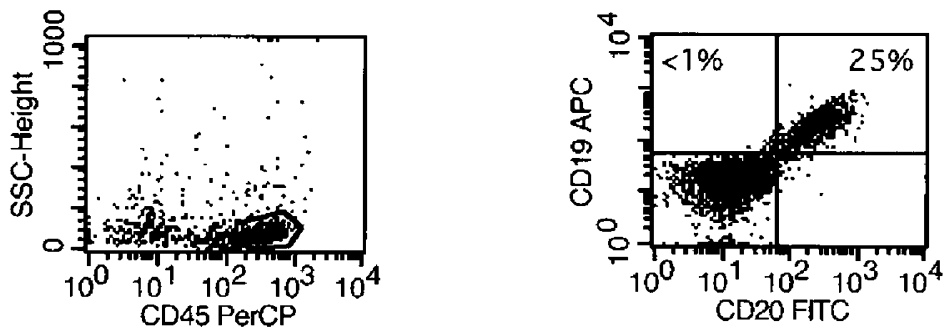

Figure 4 (a) Expression of plasma cell markers on spleen lymphocytes. Spleen lymphocytes were analyzed by four-color flow cytometry using CD20 FITC/CD38 PE/CD45 PerCP/CD19 APC and CD20 FITC/CD138 PE /CD45 PerCP/CD19 APC antibodies. B cells were gated based on CD45 (as indicated in Figure 1) and CD19 positivity and analyzed for expression of CD20, CD38 and CD138. (b) Expression of Bcell markers by lymphocytes from peripheral blood and lymph nodes. A representative example of normal peripheral blood and lymph node is shown. Lymphocytes were gated using the CD45/SSC dot plot and studied for expression of CD19 and CD20 markers.

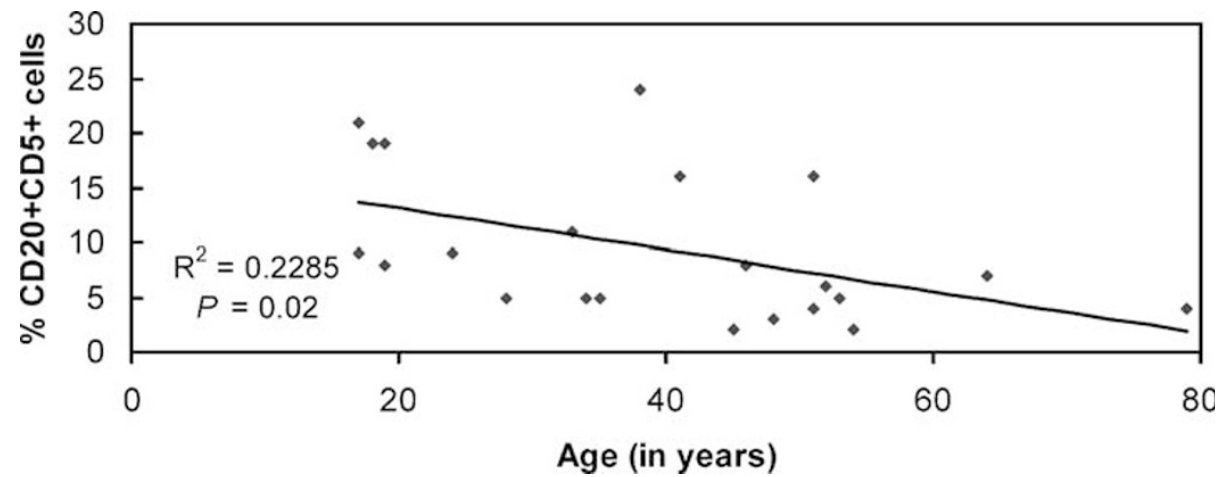

Figure 5 Distribution of individual percent values for CD5 + CD20 + cells vs age. Simple linear equation was applied to determine $P$ and $r^{2}$ values.

strongly suggest that reactive spleen displays a higher frequency of $\mathrm{T}$ cells and a lower frequency of $\mathrm{B}$ cells as compared to normal spleen.
Previous studies of normal B cells present in human peripheral blood and lymph nodes showed that pan-B cell markers CD20 and FMC7 were 

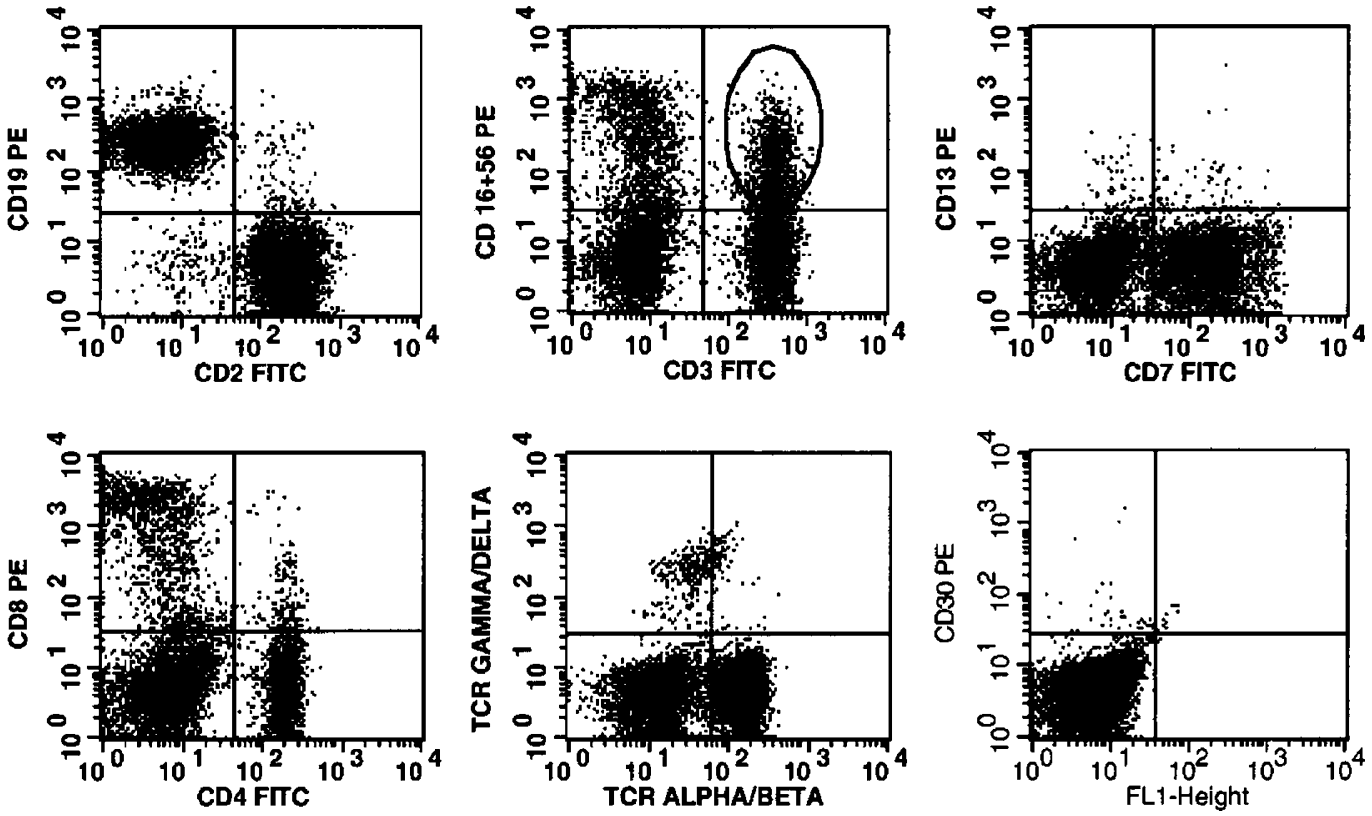

Figure 6 Immunophenotypic profile of splenic T and NK cells. Spleen lymphocytes were gated as indicated in Figure 1 and analyzed for expression of pan-T and NK markers by three-color flow cytometry. The oval highlights the NK/T cell subset (CD3 + CD16/56 +).

coexpressed by nearly all CD19-positive B cells. ${ }^{11,15}$ In contrast, we found that the expression of CD20 and FMC7 did not parallel that of CD19 and CD79a on splenic B cells. To exclude the possibility that the differences between the results of our study and those of previous reports are due to the use of different flow cytometry protocols, lymphocytes from normal peripheral blood and lymph nodes were analyzed using the same method and reagents as used for spleen cells. Our data indicate that the frequency of CD19+CD20- B cells is, indeed, higher in spleen as compared to peripheral blood or lymph nodes.

The study of Ig light chain expression on splenic B lymphocytes showed that surface Kappa and Lambda chains were expressed by CD19+CD20 + $\mathrm{B}$ cells yet were absent or expressed at very low levels by CD19 + CD20- B cells. However, cytoplasmic expression of Kappa and Lambda was found on virtually all CD19-positive cells. Furthermore, CD19 + CD20 - cells expressed high levels of CD38, and were negative for CD138. These results are consistent with the presence in the spleen of a sizable population of early plasma cells, ${ }^{19-21}$ carrying the CD45+CD19+CD38 bright, Kappa dim/ negative, Lambda dim/negative, CD20-CD138- phenotype, and accounting for about 6 and $3 \%$ of $B$ cells in normal and reactive spleen, respectively.

FMC7 antibody has been shown to recognize a CD20-related epitope, presumably derived from a multimeric CD20 complex. ${ }^{25,26}$ However, in some neoplastic lymphoproliferative disorders, the levels of FMC7 and CD20 expression show no correlation and, in fact, the pattern of FMC7 and CD23 coexpression has been found to be valuable for the accurate and reproducible classification of B-cell lymphomas. ${ }^{27}$ The results reported here regarding the expression of FMC7 on normal splenocytes provide a useful reference for the interpretation of an abnormal B-cell immunophenotype. The frequency of splenic FMC7-positive lymphocytes was found to be significantly lower than the frequency of CD19 + cells, and also lower than that of CD20positive cells. Therefore, the absence of FMC7 on subpopulations of splenic B cells should not be interpreted as an aberrant phenotype in the absence of other abnormal phenotypic features.

A significant population of CD20-positive B cells from the spleen was found to express CD5, namely 8 and $11 \%$ of the lymphocytes in normal and reactive spleen, respectively. Previous studies have identified the B-cell population coexpressing CD5 and CD20 antigens as the B-1a subset, and found that 2$3 \%$ of the lymphocytes from normal adult tonsils and lymph nodes display this phenotype. ${ }^{28}$ During fetal life and childhood, however, CD5 + B cells were shown to represent a major B-cell subset, accounting for $40-60 \%$ of fetal splenic B cells. ${ }^{29,30}$ Although the ontogeny and function of these cells are not well understood, it is assumed that they provide an innate immune defense early in life by producing antibodies against ubiquitous bacteria. ${ }^{31}$ Our present study indicates that the frequency of splenic CD20 + CD5 + cells decreases with age. This finding is in accordance with previous data showing the same type of correlation between the frequency of CD5 + B cells from peripheral blood and age. ${ }^{32}$

The level of expression of the CD5 marker observed on normal splenic B cells is lower than that seen on $\mathrm{T}$ cells. Thus, in most cases flow 
cytometry permits the distinction between normal B cells, which show dim expression of CD5, and leukemic B cells (eg chronic lymphocytic leukemia (CLL) and mantle cell lymphoma), ${ }^{4,15}$ which typically appear as a well-defined cluster of CD20/CD5 double-positive cells, expressing CD5 at levels close to those found on T cells.

Similar to the CD5 marker, CD23 is a critical marker for the diagnosis of B-cell disorders, allowing the distinction between CLL and mantle cell lymphoma. ${ }^{4}$ In contrast to CD5 pattern of expression, CD23 shows increased expression with age, from $35 \%$ in cord blood to over $60 \%$ of B cells in adult blood. ${ }^{15}$ We found that a relatively large cell population expressed CD23, representing 34 and $43 \%$ of the lymphocytes from normal and reactive spleen, respectively. However, no significant correlation between expression of the CD23 marker and age could be established for spleen lymphocytes.

The study of T-cell populations present in the human spleen revealed that the CD4/CD8 ratio is on the average 1.2:1, similar to the ratio found in lymph nodes and bone marrow yet lower than in peripheral blood, where it usually exceeds $2: 1 .^{11}$ The frequency of $\gamma / \delta$ TCR-positive cells was $6 \%$ and $11 \%$ of all CD3 positive $\mathrm{T}$ cells in normal and reactive spleen, respectively. These values may appear much lower than those previously reported by studies which showed that $\gamma / \delta$ TCR-positive cells represent about $17 \%$ of all CD3 positive cells in the red pulp of the spleen. ${ }^{33-35}$ However, the same studies reported that, in periarteriolar sheaths, $\gamma / \delta$ TCR expressing cells were rare. Thus, the apparent discrepancy between our results and previously reported data is caused by the different approaches used to analyze and report the frequency of $\gamma / \delta$ TCR-positive cells. While previous studies used immunohistochemistry and reported a relatively high frequency of $\gamma / \delta$ TCRpositive cells within the red pulp area of the spleen, our study used flow cytometry and reported the frequency of this subset within whole spleen.

A sizable proportion of splenic $\mathrm{T}$ cells exhibit dual expression of $\mathrm{T}$ and $\mathrm{NK}$ cell markers, a phenotypic signature of NK/T cells. ${ }^{36}$ This T-cell subset has been intensively studied as a possible bridge between innate and adaptive immunity. The proposed role of $\mathrm{NK} / \mathrm{T}$ cells in immune responses ranges from suppression of autoimmunity to tumor rejection. ${ }^{36,37}$ While in peripheral blood NK/T cells account for less than $6 \%$ of CD3-positive lymphocytes, our study shows that about $16 \%$ of the CD3positive cells present in the spleen express CD16/56 antigen(s). A relatively high frequency of these cells has been also reported in the liver, where up to $55 \%$ (mean 27\%) of CD3-positive lymphocytes express NK markers. ${ }^{37}$ These findings support the concept that spleen and liver lymphocytes have important immunoregulatory function.

Using a clear description of biological and technical aspects of sample analysis by flow cytometry, our study provides a frame of reference for B,
$\mathrm{T}$, and NK subsets present in normal and reactive spleen. These results will be useful for flow cytometry assessment of spleen specimens obtained following fine-needle aspiration or splenectomy, facilitating an accurate diagnosis of lymphoid malignancies of the spleen.

\section{Acknowledgement}

This work was supported by NIH grant RO1AI25210-16.

\section{References}

1 Borowitz MJ, Bray R, Gascoyne R, et al. US-Canadian consensus recommendations on the immunophenotypic analysis of hematologic neoplasia by flow cytometry: data analysis and interpretation. Cytometry 1997;30:236-244.

2 Dunphy CH. Contribution of flow cytometry immunophenotyping to the evaluation of tissues with suspected lymphoma. Cytometry 2000;42:296-306.

3 D'Arena G, Musto P, Cascavilla N, et al. Quantitative flow cytometry for the differential diagnosis of leukemic B cell chronic lymphoproliferative disorders. Am J Hematol 2000;64:275-281.

4 DiGiuseppe JA, Borowitz MJ. Clinical utility of flow cytometry in the chronic lymphoid leukemias. Semin Oncol 1998;25:6-10.

5 Imashuku S, Obayashi M, Hosoi G, et al. Splenectomy in haemophagocytic lymphohistiocytosis: report of histopathological changes with CD19+ B-cell depletion and therapeutic results. Br J Haematol 2000;108: 505-510.

6 Lucio P, Parreira A, van den Beemd MW, et al. Flow cytometric analysis of normal B cell differentiation: a frame of reference for the detection of minimal residual disease in precursor B-ALL. Leukemia 1999;13: 419-427.

7 Cornfield DB, Mitchell Nelson DM, Rimsza LM, et al. The diagnosis of hairy cell leukemia can be established by flow cytometric analysis of peripheral blood, even in patients with low levels of circulating malignant cells. Am J Hematol 2001;67:223-226.

8 Herzenberg LA, Parks D, Sahaf B, et al. The history and future of the fluorescence activated cell sorter and flow cytometry: a view from Stanford. Clin Chem 2002;48: 1819-1827.

9 Schnizlein-Bick CT, Mandy FF, O’Gorman RG, et al. Use of CD45 gating in three and four-color flow cytometric immunophenotyping. Cytometry 2002; 50:46-52.

10 Bergeron M, Nicholson JK, Phaneuf S, et al. Selection of lymphocyte gating protocol has an impact on the level of reliability of T-cell subsets in aging specimens. Cytometry 2002;50:53-61.

11 McCoy Jr JP, Overton WR. Quality control in flow cytometry for diagnostic pathology: II. A conspectus of reference ranges for lymphocyte immunophenotyping. Cytometry 1994;18:129-139.

12 Kotylo P, Fineberg NS, Freeman KS, et al. Reference ranges for lymphocyte subsets in pediatric patients. Am J Clin Pathol 1993;100:111-115. 
13 Bryan CF, Eastman PJ, Conner JB, et al. Clinical utility of a lymph node normal range obtained by flow cytometry. Ann NY Acad Sci 1993;677:404-406.

14 Ginaldi L, DeMartinis M, D’Ostilio A, et al. Changes in the expression of surface receptors on lymphocyte subsets in the elderly: quantitative flow cytometric analysis. Am J Hematol 2001;67:63-72.

15 Deneys V, Mazzon AM, Marques JL, et al. Reference values for peripheral blood B lymphocyte subpopulations: a basis for multiparametric immunophenotyping of abnormal lymphocytes. J Immunol Meth 2001;253: 23-36.

16 Fukushima PI, Nguyen PK, O’Grady P, et al. Flow cytometric analysis of kappa and lambda light chain expression in evaluation of specimens for B-cell neoplasia. Cytometry 1996;26:243-252.

17 Dixon WJ. BMDP Statistical Software New System 2.0 Professional. University of California Press: Berkeley, 1992.

18 Braylan RC, Orfao A, Borowitz MJ, et al. Optimal number of reagents required to evaluate hematolymphoid neoplasias: results of an international consensus meeting. Cytometry 2001;15:23-27.

19 Harada Y, Kawano MM, Huang N, et al. Identification of early plasma cells in peripheral blood and their clinical significance. Br J Haematol 1996;92:184-191.

20 Tarte K, Zhan F, De Vos J, et al. Gene expression profiling of plasma cells and plasmablasts: towards a better understanding of the late stages of B-cell differentiation. Blood 2003;102:592-600.

21 Medina F, Segundo C, Campos-Caro A, et al. The heterogeneity shown by human plasma cells from tonsil, blood, and bone marrow reveals graded stages of increasing maturity, but local profiles of adhesion molecule expression. Blood 2002;99:2154-2161.

22 Hayakawa K, Hardy RR. Development and function of B-1 cells. Curr Opin Immunol 2000;12:346-353.

23 deLeon ED, Alkan S, Huang JC, et al. Usefulness of an immunohistochemical panel in paraffin-embedded tissues for the differentiation of B-cell non-Hodgkin's lymphomas of small lymphocytes. Mod Pathol 1998;11:1046-1051.

24 Stein H, Foss HD, Durkop H, et al. CD30(+) anaplastic large cell lymphoma: a review of its histopathologic, genetic and clinical features. Blood 2000;96: 3681-3695.

25 Serke S, Schwaner I, Yordanova M, Szczepek A, Huhn D. Monoclonal FMC7 detects a conformational epitope on the CD20 molecule: evidence from phenotyping after rituxan therapy and transfectant cell analyses. Cytometry 2001;46:98-104.

26 Hubl W, Iturraspe J, Braylan RC. FMC7 antigen expression on normal and malignant B-cells can be predicted by expression of CD20. Cytometry 1998;15:71-74.

27 Ahmad E, Garcia D, Davis BH. Clinical utility of CD23 and FMC7 antigen coexistent expression in B-cell lymphoproliferative disorder subclassification. Cytometry 2002;50:1-7.

28 Caligaris-Cappio F, Gobbi M, Bofill M, et al. Infrequent normal B lymphocytes express features of B-chronic lymphocytic leukemia. J Exp Med 1982; 155:623-628.

29 Matsumura T, Kametani Y, Ando K, et al. Functional CD5+ B cells develop predominantly in the spleen of NOD/SCID/gC ${ }^{\text {null }}$ (NOG) mice transplanted either with human umbilical cord blood, bone marrow or mobilized peripheral blood CD34+ cells. Exp Hematol 2003;31:789-797.

30 Antin JH, Emerson SG, Martin P, et al. Leu-1+ (CD5+) B cells. A major lymphoid subpopulation in human fetal spleen: phenotypic and functional studies. J Immunol 1986;136:505-510.

31 Stall AM, Wells SM, Lam K-P. B-1 cells: unique origins and functions. Semin Immunol 1996;8:45-59.

32 Hoffkes HG, Schmidtke G, Uppenkamp M, et al. Multiparametric immunophenotyping of $\mathrm{B}$ cells in peripheral blood of healthy adults by flow cytometry. Clin Diagn Lab Immunol 1996;3:30-36.

33 Bordessoule D, Gaulard P, Mason DY. Preferential localization of human lymphocytes bearing gamma delta $\mathrm{T}$ cell receptors to the red pulp of the spleen. J Clin Pathol 1990;43:461-464.

34 Falini B, Flenghi L, Pileri S, et al. Distribution of $\mathrm{T}$ cells bearing different forms of the T cell receptor $\gamma / \delta$ in normal and pathological human tissues. J Immunol 1989;143:2480-2488.

35 Kabelitz D, Wesch D, Hinz T. Gamma delta T cells, their $\mathrm{T}$ cell receptor usage and role in human diseases. Springer Semin Immunopathol 1999;21:55-75.

36 Godfrey DI, Hammond KJ, Poulton LD, et al. NKT cells: facts, functions and fallacies. Immunol Today 2000; 21:573-583.

37 Norris S, Doherty DG, Collins C, et al. Natural T cells in the human liver: cytotoxic lymphocytes with dual $\mathrm{T}$ cell and natural killer cell phenotype and function are phenotypically heterogeneous and include Valpha24JalphaQ and gamma delta T cell receptor bearing cells. Hum Immunol 1999;60:20-31. 